\title{
Controlled Experiments and Optimized Theory of Absorption Spectra of Li Metal and Salts
}

\author{
Subhayan Roychoudhury ${ }^{\S \dagger}$, Zengqing Zhuo ${ }^{\S \dagger}$, Ruimin Qiao ${ }^{\S \dagger}$, Liwen Wan`, Yufeng Liang ${ }^{\#}$, Feng \\ Pan $^{*}$, Yi-de Chuang ${ }^{{ }^{*}}$, David Prendergast ${ }^{\# *}$, Wanli Yang ${ }^{{ }^{*}}$ \\ $\S$ Advanced Light Source, Lawrence Berkeley National Laboratory, 1 Cyclotron Road, Berkeley CA 94720, \\ USA \\ "Lawrence Livermore National Laboratory, 7000 East Avenue, Livermore, California 94550, USA \\ ${ }^{\star}$ School of Advanced Materials, Peking University Shenzhen Graduate School, Shenzhen 518055, China \\ \#The Molecular Foundry, Lawrence Berkeley National Laboratory, 1 Cyclotron Road, Berkeley CA \\ 94720, USA
}

\begin{abstract}
Investigations of Li metal and ionic compounds through experimental and theoretical spectroscopy has been of tremendous interest due to their prospective applications in Li-metal and Li-ion batteries. Li $K$-edge soft X-ray absorption spectroscopy (sXAS) provides the most direct spectroscopic characterization; unfortunately, due to the low core-level energy and the highly reactive surface, Li- $K$ sXAS of Li metal has been extremely challenging, as evidenced by many controversial reports. Here, through controlled and ultra-high energy resolution experiments of two kinds of in-situ prepared samples, we report the intrinsic Li- $K$ sXAS of Li-metal that displays a prominent leading peak, which has never been revealed before. Furthermore, theoretical simulations show that the Li- $K$ sXAS is strongly affected by the response of the valence electrons to the core-hole due to the low number of valence electrons in Li. We successfully reproduce the Li- $K$ sXAS by state-of-the-art calculations with considerations of a number of relevant parameters such as temperature, resolution, and especially contributions from transitions which are forbidden in the so-called single-particle treatment. Such a comparative experimental and theoretical investigation is further extended to a series of Li ionic compounds, which highlight the importance of considering the total and single-particle energies for obtaining an accurate alignment of the spectra. Our work provides the first reliable Li- $K$ sXAS of Li metal surface with advanced theoretical calculations. The experimental and theoretical results provide a critical benchmark for studying Li surface chemistry in both metallic and ionic states.
\end{abstract}

Keywords: X-ray absorption spectroscopy; Theory of spectroscopy; Lithium Metal; Lithium Salt; Lithium K-edge spectroscopy. 


\section{INTRODUCTION:}

Lithium-based electrochemical devices for energy storage, i.e., batteries, have been ubiquitous in modern life since its commercialization in early $1990 \mathrm{~s}^{1}$. However, in spite of several decades of extensive efforts, the holy grail of high-energy lithium battery based on Li metal anode is yet to be achieved. The challenge of utilizing Li metal electrode remains formidable in both the practical optimizations and fundamental understandings ${ }^{2,3}$. Additionally, electrolyte and its associated solid-electrolyte interphase (SEI) and cathode-electrolyte interphase (CEI) remain the least understood but critical for the battery performance ${ }^{4,5}$. Fundamentally, many limiting factors in battery operation are associated with the Li-ion diffusion, which is extremely difficult to track and could only be peered into through indirect structural probes coupled with theoretical calculations ${ }^{6}$. Therefore, the understandings and optimizations of many battery operation issues could benefit from a direct probe of $\mathrm{Li}$ in its metallic and ionic states with elemental and chemical sensitivities.

The technical challenges for a direct probe of Li chemistry stem from many intrinsic characters. First, as an Alkali metal, Li metal is highly reactive, so its surface is unstable under ambient environment. As revealed by the controlled experiments below, even in ultra-high vacuum environment, the surface of Li metal could still evolve quickly. Second, as a popular technique, soft X-ray absorption spectroscopy (sXAS) has long been employed to study battery materials for many other elements, especially through its photon-in-photon-out (PIPO) mode with relatively bulk-sensitivity ${ }^{7}$. However, the only core-level excitation of Li, the $K$-edge electron excitation from $l s$ to $2 p$, falls into a very low energy range around only $55 \mathrm{eV}$. This low energy naturally leads to a shallow probe depth even in the sXAS PIPO mode. Third, for the ionic compounds, it has been observed that soft X-rays around the Li- $K$ edge could introduce radiation damage effects ${ }^{8}$. So experiments with controlled surface preparation and irradiation are critical for obtaining reliable data. Fourth, due to these technical challenges, compared with a large number of sXAS reports of other elements, e.g., $\mathrm{O}$ and transition metals, there are only a handful of literature with experimental results of $\mathrm{Li}-K \mathrm{sXAS}{ }^{8-15}$. $\mathrm{Li}-K \mathrm{sXAS}$ was occasionally reported in battery electrode studies, but due to the lack of benchmark and fundamental understandings, they were merely utilized as just supplementary indications on the amount of Li during the lithiation/delithiation process $^{16}$. Even worse, the spectra of Li metal have been very different from each other in different reports, and contradict the findings by other techniques such as EELS and hard X-ray Raman ${ }^{9-}$ $11,17-19$.

On the theory front, Li-K sXAS of various ionic compounds has been computed ${ }^{20,21}$ with the help of Kohn-Sham (KS) Density Functional Theory (DFT) ${ }^{22,23}$ and many-body perturbation theory ${ }^{24}$. Researchers also simulated $\mathrm{Li}-K$ sXAS of metallic $\mathrm{Li}$ using wave-function based single particle techniques and perturbative treatments ${ }^{19,25}$. However, the precision and validity of the theoretical calculations suffer the lack of reliable experimental results, so it has been hard to achieve a quantitative level of comparisons between the theory and experiments. Therefore, establishing the benchmark of reliable experimental data and developing theoretical calculations of $\mathrm{Li}-\mathrm{K}$ are equally important towards the direct probe of Li chemistry of the aforementioned electrode materials and interfaces in batteries.

In this paper, controlled experiments were carried out by employing two kinds of in-situ Lithium metal sample preparations, evaporation and cleaving, directly in the ultra-high vacuum (UHV) with ultra-high experimental resolution. Aging and radiation effects are tested separately. We show 
that the surface of Li metal is highly reactive even in $1.4 \times 10^{-10}$ Torr UHV environment towards $\mathrm{Li}_{2} \mathrm{O}$. Samples prepared by in-situ Li evaporation in UHV display the degradation signature right after the preparation, likely due to the immediate reaction at the high temperature of the evaporation process. Comparative experiments on three sets of samples show that the surface prepared through in-situ cleaving finally rules out all degradation possibilities, and allows the collection of the intrinsic Li- $K$ spectrum of Li metal. Li- $K$ sXAS of Li metal displays a prominent leading peak at the absorption edge that has never been found before. Our theoretical calculations show that, due to the low atomic number of $\mathrm{Li}$, the poorly screened core-hole strongly affects the unoccupied orbitals. An accurate calculation requires the explicit consideration of the orbital relaxation of all the valence electrons in response to the core-hole creation, which we simulate with the state-of-the-art Many-body X-ray Absorption Spectroscopy (MBXAS) method ${ }^{26,27}$. We successfully explore the effects from temperature, resolution, and contributions from transitions which are forbidden within the traditional single-particle framework and thereby achieve a successful reproduction of the experimental data and explain the previous controversial reports. We further investigate a series of Li ionic compounds based on such a theoretical approach. The simulations show that, in order to obtain accurate spectral alignment among different compounds, each spectrum must be rigidly shifted by a term containing total as well as single-particle KS energies computed using hybrid exchange-correlation (XC) functional. Our work provides the first reliable dataset of $\mathrm{Li}-K$ sXAS of $\mathrm{Li}$ metal through the carefully controlled experiments, and establishes the framework for optimized theoretical calculations to obtain a simulation of $\mathrm{Li}-K$ sXAS spectroscopy with quantitative accuracy in terms of lineshape and alignment.

\section{EXPERIMENTAL AND THEORETICAL SECTION}

$\mathrm{Li}-K \mathrm{sXAS}$ was performed at the ultra-high energy resolution Beamline 4.0.3 (MERLIN) of the Advanced Light Source at Lawrence Berkeley National Laboratory. The beamline covers energies ranging from $10 \mathrm{eV}$ to $\sim 150 \mathrm{eV}$, which is ideal for $\mathrm{Li} K$-edge, with a photon flux of $10^{11} / \mathrm{sec}$. The experimental energy resolution is better than $10 \mathrm{meV}$. Due to such a high instrumental resolution, it is expected that the finite peak width of the spectrum is predominantly a consequence of the thermal effects and the finite lifetimes of the Li quasiparticle orbitals, including the core-hole and the conduction orbitals. Both total fluorescence yield (TFY) and the total electron yield (TEY) signals are collected; however, we note that due to the very low photon energy of Li- $K$ around 50$60 \mathrm{eV}$, the attenuation of the X-ray photons is around $50 \mathrm{~nm}$ [Supplementary FIG.S4], so even for the TFY collected in the reflection mode (photon-in-photon-out), majority of the signals are from the surface $10-20 \mathrm{~nm}$, roughly at the same scale as that of TEY. This is confirmed by the similar Li- $K$ TEY and TFY lineshape of the Li-metal samples, as shown below. For Li salts, due to their highly insulating property, TEY is not an ideal probe, so overall, we focus on TFY signals in this work.

Two different in-situ sample preparation methods are used to prepare the Li metal samples: cleaving a thick piece of Li metal and Li evaporation. The in-situ cleaving of the Li metal surface was done by installing a cleaned blade through a motion feedthrough into the UHV $\left(1.4 \times 10^{-10}\right.$ Torr) chamber, which is used to cut/scratch through the soft Li metal to expose a fresh surface. Evaporated Li metal surfaces are prepared by supplying a controlled current through a SAES Li metal dispenser, which has been well degassed before the Li evaporation. We note SAES alkali metal dispensers are typical alkali metal sources in scientific studies for controlled Alkali-metal evaporation in $\mathrm{UHV}^{28}$. Surprisingly, the two kinds of in-situ prepared samples display clear 
differences (FIG. $1 \&$ 2), which provides the chance to reveal the highly reactive Li metal surface even in $10^{-10}$ Torr ultra-high vacuum (UHV) and to clarify the discrepancy in previous reports.

On the theory front, we simulate the spectra with the MBXAS formalism which approximates the initial (final) state as a Slater determinant composed of valence KS orbitals obtained in the absence (presence) of a core-hole. As discussed in the next section, with such explicit consideration of the relaxation of valence electrons, MBXAS is able to show that electronic transitions which are forbidden in the conventional single-particle approach can actually have significant amplitude. For simulating room temperature effects, we use MBXAS in conjunction with ab-initio molecular dynamics (MD) which leads to an overall smoothening of the spectrum, in accordance with the experimental results. Finally, in order to obtain an accurate alignment among the spectra of different materials, we propose a scheme based on a combined consideration of the total and singleparticle energies obtained using a non-local XC functional.

\section{RESULTS AND DISCUSSIONS}

Li-K spectroscopy of Li metal: FIG.1(a) displays the sXAS recorded right after the cleaved metal surface is exposed at a regular interval of 10 minutes each for 15 hours. The bottommost spectrum corresponds to the freshly prepared sample, which shows a strong peak at $55.6 \mathrm{eV}$, as can be seen in the amplified display in FIG.1(b). The presence of this peak is generally consistent with several previous reports based on bulk-sensitive techniques ${ }^{11,17,25,29}$, however, with a much stronger leading peak here. Moving from the bottom to the top, the 15 thick spectra indicate the lineshape evolution every one hour. Obvious change is observed after 20 minutes, when the profound 55.6 $\mathrm{eV}$ feature begins to diminish and the features at $58.4 \mathrm{eV}$ and $62.9 \mathrm{eV}$ start intensifying. The latter two resemble the peaks of a $\mathrm{Li}_{2} \mathrm{O}$ spectrum, as shown by the reference spectra on top. After about 4 hours in the UHV chamber, evident features from $\mathrm{Li}_{2} \mathrm{O}$ start to dominate the spectrum and the signatures of the freshly prepared Li sample get obscured. The results here clearly show that, even at $10^{-10}$ Torr UHV, Li metal surface still degradants very fast. This naturally explains why experiments with Li metal samples loaded from inert gas bags or even air reported very different results ${ }^{9,10}$. Furthermore, the formation of $\mathrm{Li}_{2} \mathrm{O}$ is intriguing, because essentially all $\mathrm{O}_{2}$ molecules are removed in the UHV environment, and residual gas molecules of UHV at $10^{-10}$ Torr scale are water and others with light molecular weight. The dominating $\mathrm{Li}_{2} \mathrm{O}$ signals as the degradation product of Li metal under UHV implies a multi-step process instead of a direct Li oxidation.

In order to rule out irradiation effects for such a spectral evolution, we collect the in-situ spectrum of a separate sample, prepared and stored under similar conditions in the UHV for 5 hours without any exposure to the X-ray beam. As shown in the inset of FIG.1(a), this spectrum is essentially identical to that collected after 5 hours from the sample which has been exposed to X-rays. This supports our inference that the disappearance of the $55.6 \mathrm{eV}$ peak upon time is due to surface degradation even under UHV, not due to irradiation.

FIG.1(b) shows the amplified spectra, collected in both the TFY and TEY modes, for Li metal prepared by cleaving (same spectrum as the Fresh spectrum in FIG.1a), compared with the spectra collected from samples prepared by in-situ evaporation (blue). In sharp contrast, the evaporated Li 
metal displays evident signatures of the $\mathrm{Li}_{2} \mathrm{O}$ spectrum on the freshly prepared sample, indicating the sample surface already degraded right after the in-situ evaporation.

To conclude the intrinsic Li-K sXAS of Li metal, we further tested samples prepared in-situ by both the cleaving and evaporation on exactly the same piece of Li metal, as shown in FIG.2 with the sample preparation sequence from the bottom to the top. First the cleaved Li metal sample displays the strong $55.6 \mathrm{eV}$ leading peak, as found above. Second, the sample is left in UHV for about 3 hours, which displays the clear signature of the degradation product of $\mathrm{Li}_{2} \mathrm{O}$. Third, we evaporate lithium onto the surface of the sample with the evaporation time of 10,11 and 15 minutes. The intensity of the $\mathrm{Li}_{2} \mathrm{O}$ features show that the surface is getting cleaner towards $\mathrm{Li}$ metal upon evaporation time until more than 10 minutes, and further Lithium evaporation longer than 11 minutes does not improve the overall spectral lineshape. Fourth, the red spectrum on top was collected on the same sample but with the surface cleaved again with the blade. The TEY and TFY of Li metal samples have been similar due to the same level of probe depth as discussed above.

Therefore, these three sets of controlled experiments consistently suggest that Li metal surface is highly reactive even under UHV conditions. Because in-situ evaporation introduces a much higher local temperature around the sample, it is not surprising that a large amount of freshly evaporated Li already degraded right after the deposition. Therefore, intrinsic Li metal $K$-edge sXAS could only be obtained within a short time through in-situ cleaving sample preparation under UHV conditions. This also naturally explained the contradictory results in previous reports that have not been performed with such a controlled sample preparation ${ }^{9-11}$. More importantly, the in-situ cleaved samples have displayed a very robust and reproducible strong $55.6 \mathrm{eV}$ peak that has not been found before with even bulk-sensitive techniques ${ }^{18,19,25}$. It is thus critical to interpret this intrinsic Li-metal Li- $K$ lineshape.

\section{Theoretical calculations of Li-K sXAS of Li metal}

In order to reproduce and understand the striking lineshape of the intrinsic Li metal Li- $K$ spectrum, we have explored different parameters and successfully developed the theoretical calculation of the Li K-edge sXAS based on DFT. Details of the computational parameters are presented in the Supplementary Information. The dipole transition matrix element for electronic excitation from an initial state $\left|\Psi_{i}\right\rangle$ to a final state $\left|\Psi_{f}\right\rangle$ is given by $\left\langle\Psi_{f}|\widehat{\boldsymbol{O}}| \Psi_{i}\right\rangle$ where, $\widehat{\boldsymbol{O}}$ is the many-electron dipole transition operator. In the single-particle initial state approximation, one approximates this term by

$$
\left\langle\Psi_{f}|\widehat{O}| \Psi_{i}\right\rangle \approx S\left\langle\phi_{c}|\hat{o}| \phi_{\text {core }}\right\rangle, \quad \quad E q .1
$$

where $\phi_{\text {core }}$ is the single-particle wave-function of the core electron, $\phi_{c}$ is a Kohn-Sham (KS) unoccupied (i.e., conduction band) orbital obtained with the initial (ground) state DFT calculation run in absence of the core-hole, $\hat{o}$ is now the single-particle dipole transition operator, and $\mathrm{S}$ is a constant.

The simulation of the Li K-edge spectrum of bulk Li metal using this treatment is presented at the bottom of FIG.3. The final state Full Core-hole $(F C H)$ spectrum, where $\phi_{c}$ in Eq. 1 is replaced by 
$\tilde{\phi}_{c}$, a final-state conduction orbital evaluated in presence of the core-hole, is shown above it in the same figure. The stark difference between the initial-state and the $F C H$ spectra stems from the fact that, due to the low number of valence electrons in $\mathrm{Li}$, the core-hole is poorly screened and consequently the unoccupied orbitals obtained in presence and absence of the core-hole are very different. In fact, the presence of the core-hole generates a set of localized orbitals trapped in the electrostatic potential of the excited core, which have significant overlap with the core orbital and result in higher intensity transitions in the spectrum.

The blue spectrum labeled "MBXAS (bulk)" is obtained with the state-of-the-art MBXAS method, which calculates the matrix element $\left\langle\Psi_{f}|\widehat{\boldsymbol{O}}| \Psi_{i}\right\rangle$ by approximating the many-body wavefunction $\Psi_{f}\left(\Psi_{i}\right)$ by a Slater determinant composed of KS orbitals computed in presence (absence) of the core-hole. Unlike the FCH spectrum, the MBXAS spectrum replicates a key feature of the experimental plot: the intensity of the first narrow peak is higher than that of the broad region at higher energy. Many of the low-energy transitions which have negligible probability in the singleparticle treatment, have appreciable probability in the MBXAS treatment. FIG.4 (a), (b) show the isovalue plots of two unoccupied KS orbitals, transitions to which contribute significantly to the first peak intensity in MBXAS. While the orbital in panel (a) is a p-type orbital centered on the core-excited atom, and is therefore representative of a standard dipole-allowed transition in the FCH treatment, the orbital in panel (b) has negligible presence on the core-excited atoms and consequently, the associated excitation does not have any significant FCH transition-probability. However, the latter excitation has a substantial probability in the MBXAS method, which computes the transition matrix element as

$$
\left\langle\Psi_{f}|\widehat{o}| \Psi_{i}\right\rangle=\sum_{c}^{\text {empty }}\left\langle\Psi_{f} \mid \Psi_{i}^{c}\right\rangle\left\langle\phi_{c}|\hat{\boldsymbol{o}}| \phi_{\text {core }}\right\rangle
$$

where $\left|\Psi_{i}^{c}\right\rangle$ represents an excited state (to orbital $c$ ) constructed with initial-state orbitals, which is represented by a Slater determinant composed of the occupied initial-state KS orbitals along with $\phi_{c}$. The term $\left\langle\Psi_{\mathrm{f}} \mid \Psi_{i}^{c}\right\rangle$, a matrix element between two Slater determinants built from orbitals of different self-consistent fields (final and initial state), can be re-expressed as the complex conjugate of a determinant composed of the overlap matrix elements between the final and initial state orbitals (See Supplementary Information for more details). A heat map showing such orbital overlaps is shown in FIG.4(c). The presence of significant off-diagonal elements in this overlap matrix indicates the possibility that even if, for a certain final state orbital $\widetilde{\phi_{c}}$, the transitionprobability $\left\langle\widetilde{\phi_{c \prime}}|\hat{\mathbf{o}}| \phi_{\text {core }}\right\rangle$ is zero (i.e., the transition is dipole-forbidden in the FCH picture), it is possible that for a finite number of unoccupied initial-state orbitals $\phi_{c}$, both of the terms $\left\langle\phi_{c}|\hat{\mathbf{o}}| \phi_{\text {core }}\right\rangle$ and $\left\langle\Psi_{\mathrm{f}} \mid \Psi_{i}^{c}\right\rangle$ have non-zero contributions leading to an appreciable transition probability given by the sum in Eq.2 (Note that $\Psi_{\mathrm{f}}$ is given by a Slater determinant of which $\widetilde{\phi_{c \prime}}$ is a constituent orbital.) The capability of including all these transition probabilities in our MBXAS calculations directly leads to the improved agreement with our experimental data on the strong $55.6 \mathrm{eV}$ peak intensity beyond conventional single-particle methods.

In addition to the greater intensity of the first peak provided by the MBXAS theoretical approximation, we also note that there can be more mundane origins for a difference in relative intensity of the peaks. If, instead of a Gaussian broadening of $0.1 \mathrm{eV}$, we use a much higher 
broadening of $0.6 \mathrm{eV}$, the relative intensity of the higher energy region of the MBXAS spectrum turns out to exceed that of the first peak (see Supplementary Information). Therefore, in addition to surface aging reactions, a poor instrumental resolution can result in an experimental spectrum with relatively lower first peak intensity. This naturally explains the quantitative difference between the high resolution ( $10 \mathrm{meV}$ experimental resolution) $\mathrm{Li}-K$ sXAS revealed here and the previous reports of hXRS and EELS results ${ }^{18,19,25}$.

In order to obtain a more realistic simulation of the experiment, which is performed at room temperature, we run a first-principles MD simulation on the lithium crystal supercell at $300 \mathrm{~K}$ temperature and calculate, using the MBXAS method, the average sXAS spectrum on a sample snapshot (see previous examples in Ref. $\left[{ }^{20}\right]$ ). The spectrum so obtained is labeled as "MD (bulk)" in FIG.3. Comparing this with the "MBXAS(bulk)" spectrum obtained at zero temperature, we can see that the thermal motion of the ions is responsible for the relative smoothness of the former at higher energies. The surface sensitive nature of an sXAS experiment has prompted us to run MBXAS simulations on a 12-layer (18.85 $\AA$ ) thick slab of Li atoms. In FIG.3, we have shown the computed spectra corresponding to the top layer, the $5^{\text {th }}$ layer and the $6^{\text {th }}$ (middle) layer of the Li slab. MBXAS spectra for the $5^{\text {th }}$ and the $6^{\text {th }}$ layer of the slab look almost identical, indicating a convergence above 5 layers. The spectra of these layers also replicate the crucial experimental observation that the narrow first peak has higher intensity than the high energy region. It is worth noting that the top layer spectrum blue shift to higher energies $(\sim 0.8 \mathrm{eV})$ due to the slight oxidation of the surface/undercoordinated $\mathrm{Li}$ atoms. A lower ground-state valence Lowdin population (0.8524) on a surface atom compared to one in the middle of the slab (0.9797) results in a higher binding energy for the core electron in the former. A linear combination of the layer by layer spectra may lead to an intrinsic broadening the first peak evident in the bulk spectra, due to the relative prominence of this top layer contribution.

Spectroscopy of Li-based Ionic Compounds: FIG.5 shows the experimental spectra of a series of Li-based ionic compounds, which are of interest for being crucial components of the interphases in batteries as mentioned earlier; namely $\mathrm{LiF}, \mathrm{LiCl}, \mathrm{LiOH}, \mathrm{Li}_{2} \mathrm{O}, \mathrm{Li}_{2} \mathrm{O}_{2}$ and $\mathrm{Li}_{2} \mathrm{CO}_{3}$. The spectra for the latter three compounds, which were reported in our earlier work ${ }^{8}$ exploring the effects of irradiation on Li K-edge spectroscopy, have been included here for the sake of completeness and comparison. It is clear that these $\mathrm{Li}$ ionic systems display very different lineshape with features at characteristic energy positions. FIG.6 shows the theoretical counterparts obtained with MBXAS calculations. Note that these spectra, which are simulated on the static crystal structure at zero temperature, do not incorporate the effects of thermal motion of the ions, the predominant effect of which is expected to be a further smoothening of the spectra ${ }^{20}$. In FIG S2, we also present the isovalue plots of the unoccupied orbitals, transitions to which result in the prominent peaks of the spectra according to the MBXAS treatment.

SXAS energy Alignment: In the same way that experimental measurements of sXAS require some calibration to align the spectral energies, the simulations performed in this work have their own alignment scheme. However, it is worth noting that the scheme adopted here, as in previous work ${ }^{20,30,31}$, relies only on a single experimental spectrum for calibration of all such spectra, defined by the element and the core orbital. Thereafter, all computed spectra should be predictive. This is particularly important when determining the contributions of multiple components to a 
given spectrum, since their relative alignment can affect the final spectrum, for example, the different peak position of the surface vs. bulk Li atoms of Li metal as detailed above.

In our approach we focus on the particular excited state that we model explicitly at the level of DFT self-consistent-field (SCF) calculations, which is the energetically lowest possible coreexcited state for the given element and core orbital. This state has been referred to in the literature as the $\mathrm{XCH}$ state (eXcited electron and Core Hole) ${ }^{32}$, referring to a neutral reference. $\mathrm{XCH}$ is different from the FCH (Full Core Hole) state, which refers to the non-neutral $(+1)$ ionization potential for the core electron. Using relative $\mathrm{XCH}$ total energies, we can align our calculated spectra. The energy range of the spectra themselves are defined by the corresponding KS eigenvalues from the $\mathrm{XCH} \mathrm{SCF}$, referenced to the highest occupied orbital from the $\mathrm{XCH}$ calculation (which should have the same orbital index as the conduction band minimum or the lowest unoccupied molecular orbital of the ground state). Being familiar with expected underestimation $^{33,34}$ of both the electronic band gap and band width by local and semilocal approximations to the exchange-correlation potential in DFT (such as $\mathrm{PBE}^{35}$ ), here we make use of the HSE hybrid exact-exchange functional ${ }^{36}$. For insulators with large band gaps, HSE offers more accurate band structure estimates ${ }^{37}$.

We elucidate the computational scheme with the example of LiF. The lowest possible coreexcitation energy can be calculated as $\left(E_{X C H}-E_{G S}\right)$, where $E_{G S}\left(E_{X C H}\right)$ corresponds to the totalenergy of the ground state (lowest core-excited state) calculation. However, since these two calculations employ different sets of pseudopotentials, their energies cannot be compared directly and we resort to using a relative excitation energy by referencing the appropriate isolated atom:

$$
\left(E_{X C H}-E_{G S}\right)-\left(E_{X C H}^{\text {atom }}-E_{G S}^{\text {atom }}\right), \quad E q .3
$$

where $E_{G S}^{\text {atom }}\left(E_{X C H}^{\text {atom }}\right)$ refers to the energy of a single Li atom in its ground state, i.e., lowest coreexcited state, obtained with a charge-neutral DFT calculation employing the standard pseudopotential, i.e., pseudopotential containing a core-hole. One can think of this relative excitation energy as equivalent to a formation energy difference ${ }^{30}$. In order to guarantee cancellation of systematic errors, the atomic calculations are performed within the same supercell as the full system with the same numerical parameters (plane-wave cut-off, etc.).

The electronic structure of crystalline LiF exhibits a conduction band minimum with Li $2 s$ symmetry. Unsurprisingly, the XCH state places the core-excited electron in a localized orbital with Li $2 s$ symmetry centered on the core-excited Li atom. Therefore, the $\mathrm{XCH}$ state defines a dark exciton, since $1 s \rightarrow 2 s$ transitions are dipole-forbidden. The ground state has $N$ valence electrons, and so the core-excited states have $N+1$ valence electrons. Therefore, the highest occupied orbital of the XCH state has a KS eigenvalue $\tilde{\varepsilon}_{N+1}$. Our calculations indicate that the first bright transition is to the orbital with energy $\tilde{\varepsilon}_{N+3}$. Therefore, the position of the first bright transition in $\mathrm{LiF}$ could be approximated as

$$
\left(E_{X C H}-E_{G S}\right)-\left(E_{X C H}^{\text {atom }}-E_{G S}^{\text {atom }}\right)+\left(\tilde{\varepsilon}_{N+3}-\tilde{\varepsilon}_{N+1}\right)+E_{\text {shift }} . \quad E q .4
$$


We apply this expression to align the spectra of each excited atom in all the studied materials, where the orbital index of the first bright transition must be determined in each case. The constant, $E_{\text {shift }}=56.6 \mathrm{eV}$, is added to each spectrum to align it with the experimental photon energy. It was deduced specifically to align the LiF first peak. All other energies in Eq. 4 are calculated using the HSE functional. However, the overall spectrum is still computed in its entirety using the PBE functional without any dilation-factor, because the necessary calculations of many unoccupied orbitals is currently too expensive using HSE. There is one exception to this rule: for the spectral alignment of Li metal using Eq.4, PBE functional is used exclusively, since a local/semi-local functional is better suited for metallic systems in which the properties of the valence electrons are similar to that of the uniform electron gas $^{38}$. As can be seen in FIG.5 and FIG.6, with some quantitative lineshape differences, our calculated spectra show an excellent energy value agreement with the features found in experimental data for all these Li salt compounds tested here.

\section{CONCLUSION}

In summary, we present in this paper a thorough and accurate account of experimental and theoretical investigation of Li $K$-edge sXAS of Li metal and salts. Our experimental findings indicate that, due to the highly reactive Li metal surface even in UHV, a reliable spectrum of $\mathrm{Li}$ metal could only be achieved on specifically in-situ prepared samples within a short period after the sample preparation. Additionally, theoretical calculations show that energy resolution will strongly affect the intensity of especially the first peak, leading to a modified lineshape found in other bulk sensitive techniques compared with Li- $K$ sXAS. The intrinsic Li- $K$ sXAS spectrum of Li metal with $10 \mathrm{meV}$ energy resolution displays a striking peak at $55.6 \mathrm{eV}$ with a high intensity that has never been revealed before.

This provides the opportunity to test the different parameters and effects in theoretical calculations. We show that the popular $\mathrm{FCH} / \mathrm{XCH}$ approach, which expresses the approximate transition probability in terms of the core and the conduction orbital only, is inadequate for reproducing the experimental features. The explicit response of all the valence electrons to the creation of the corehole must be taken into account through the change of the KS Slater determinant. Simulations based on MD snapshot at room temperature show that thermal motion of the ions play a crucial role in the smoothening of the spectral lineshape. Eventually, we successfully reproduced the experimental results by considering the different effects and the appreciable probabilities of nondipole transitions based on MBXAS calculations.

In addition to Li metal, we also extend our experimental and theoretical studies of a series of Libased ionic compounds involved in battery materials and devices. We further show the importance of considering total energies as well as KS eigenvalues obtained with hybrid functionals in order to obtain precise relative alignment of the spectra of different materials. This work provides a reliable data bank of the Li- $K$ spectroscopy of both Li metal and several important salts, as well as developments of theoretical calculations that could reproduce the experimental results at a quantitative level in both the lineshape and energy alignment. The results set a reliable benchmark for studying the lithium chemistry and reveal critical electronic parameters that define the chemistry of the surfaces of Li compounds. 


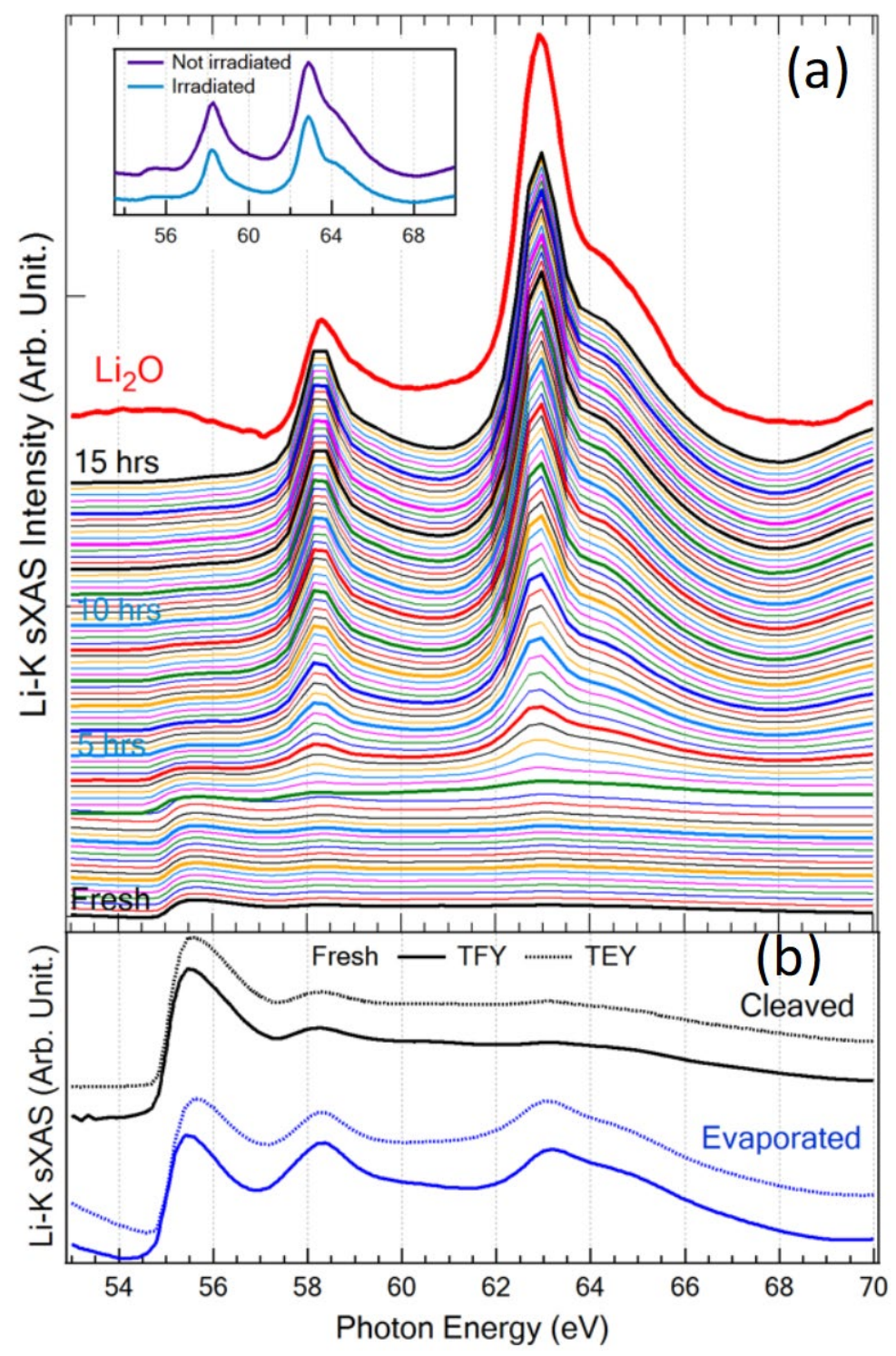

FIG.1 Soft x-ray Li- $K$ sXAS collected on lithium metal surfaces prepared in-situ in two different ways. (a) The spectral evolution of a freshly cleaved Li metal surface (bottom spectrum), then every 10 minutes in UHV of $1.4 \times 10^{-10}$ Torr. The 15 thick spectra indicate the lineshape evolution every one hour. Features from $\mathrm{Li}_{2} \mathrm{O}$ emerges after only 20 minutes in $\mathrm{HUV}$ and dominate the degraded spectral lineshape. (b) The black spectra are amplified views of the freshly cleaved Li metal surface, same as the bottom spectrum in (a). Blue spectra are collected on samples with insitu Lithium evaporation. Inset shows spectra collected on two spots with and without $\mathrm{x}$-ray exposure during the 5 hours decay period in UHV. The lineshape degrades in the same way with only small quantitative differences, indicating that the lineshape decay is from surface aging, not irradiation effect. 


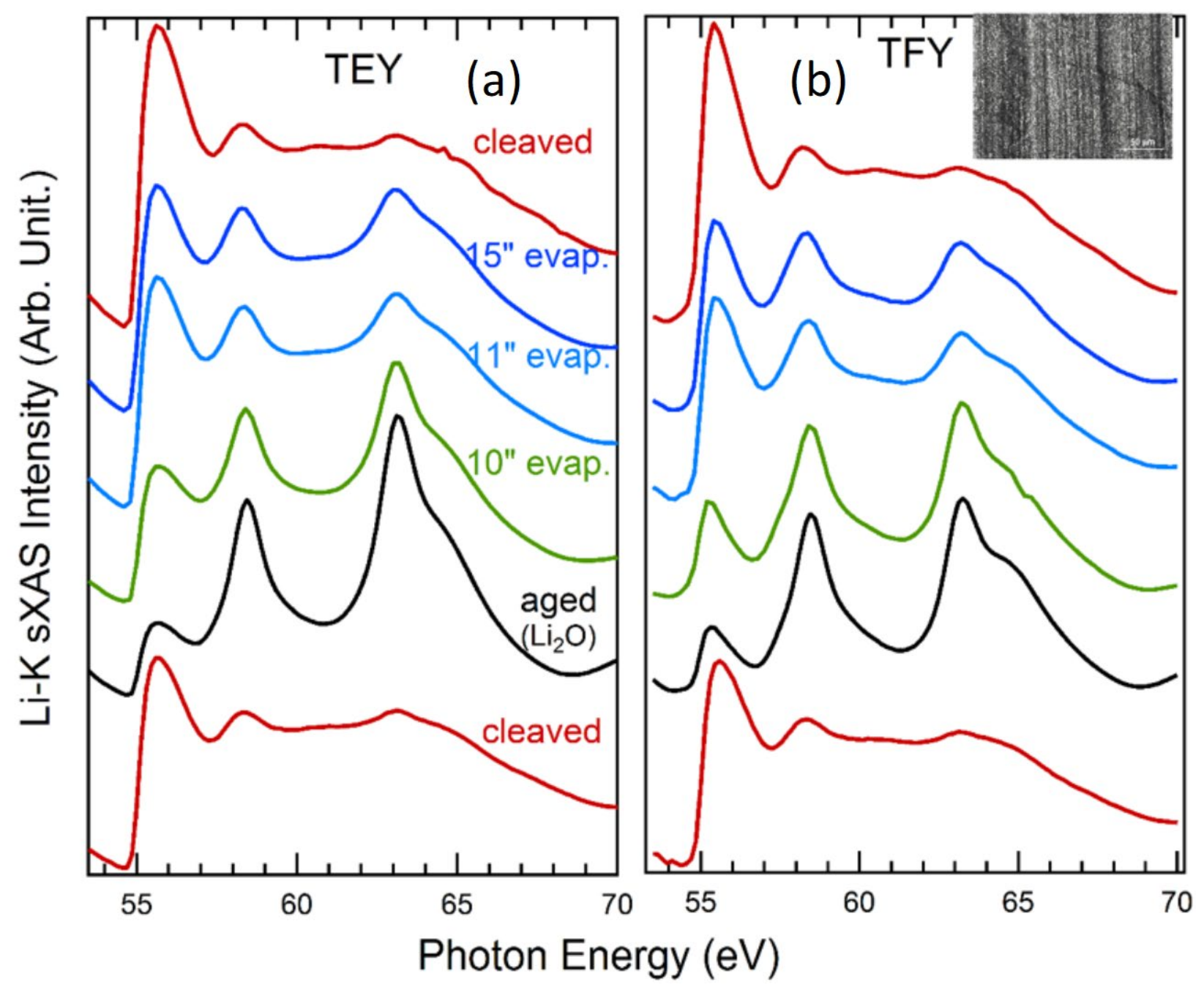

FIG.2 Li-K sXAS TEY (a) and TFY (b) spectra collected on Lithium metal prepared in the sequence of i) in-situ cleaving (bottom, red), ii) in-vacuum aged (black), iii) Lithium evaporation for 10 (green), 11 and 15 (blue) minutes, and iv) another in-situ cleaving (top, red). Further lithium evaporation does not affect the overall spectral lineshape above 11 minutes. But the in-situ cleaving is a robust method for highly reproducible $\mathrm{Li}-K$ sXAS data. Inset shows the optical image of a cleaved surface after a blade scratching. 


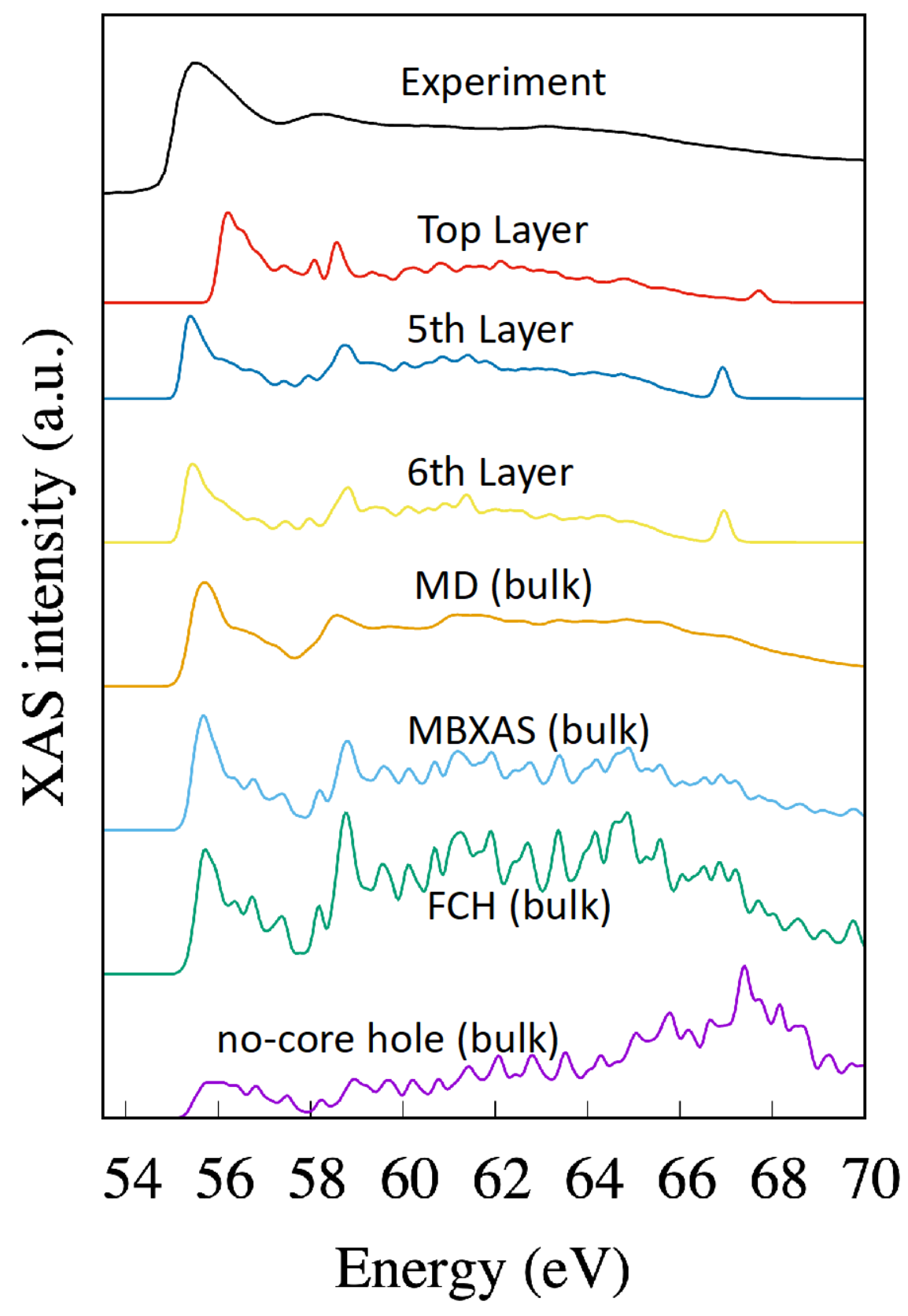

FIG.3 Plot showing X-ray absorption spectra of Li metal. From top to bottom: Experimental spectrum, simulated (with MBXAS) spectrum of the top layer of a 12-layer Li slab, spectrum of the 5th layer of the same slab, spectrum of the 6th layer of the slab, MBXAS spectrum of bulk Li crystal obtained from a MD snapshot sampled at $300 \mathrm{~K}$ temperature, MBXAS spectrum of bulk Li crystal at zero temperature, spectrum of bulk Li computed with the FCH treatment, spectrum of bulk Li simulated in absence of the core-hole. A broadening of $0.1 \mathrm{eV}$ has been applied to all the simulated spectra. 


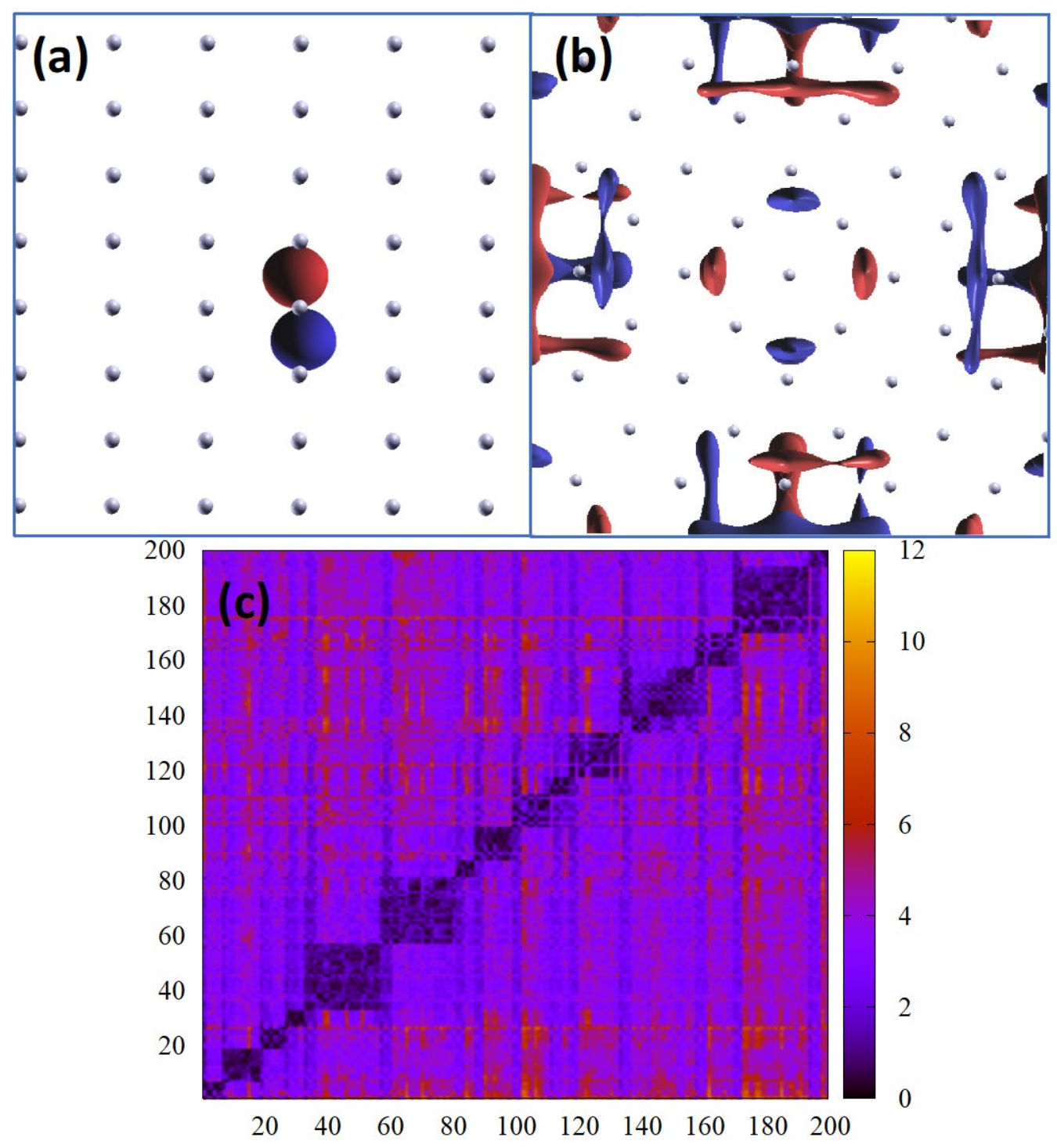

FIG.4 Panels (a) and (b) show the isovalue plots of two unoccupied final state orbitals, transitions to which generate two core-excitations having appreciable probability according to the MBXAS treatment. Panel (a), shows a p-type orbital around the core-excited atom and consequently, such a transition is dominant in the FCH approach as well. However, the orbital in panel (b) has no presence on the core-excited (central) atom and thus the $\mathrm{FCH}$ calculation shows negligible probability for this transition. However, within the MBXAS treatment, both of the transitions correspond to appreciable probability. Panel (c) shows, in the negative log scale, the overlap between the initial and the final state orbitals. A low value in this plot corresponds to a high overlap. The ground state system contains 64 up-spin valence electrons. 


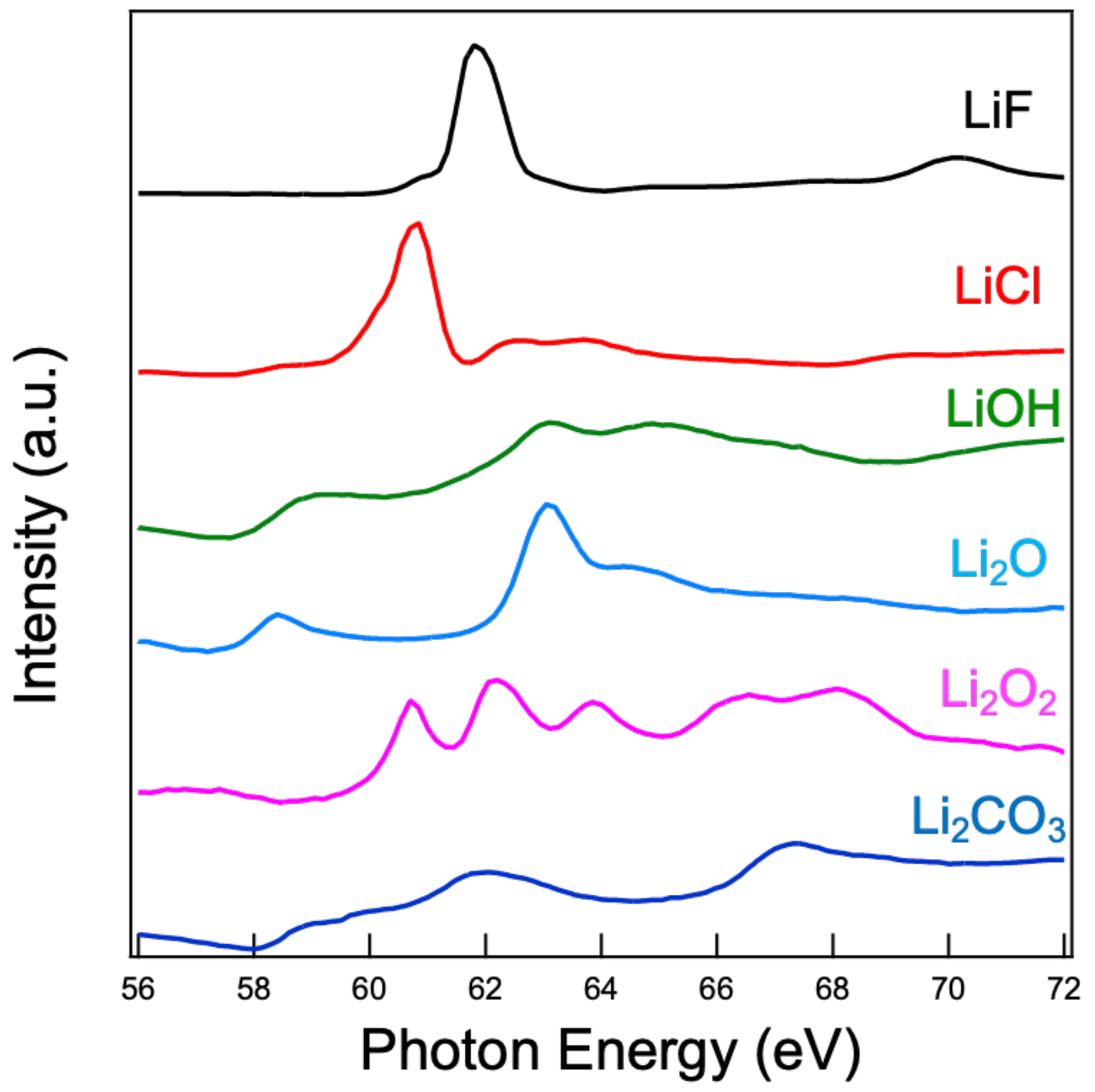

FIG.5 Experimental Li K-edge sXAS spectra of $\mathrm{LiF}, \mathrm{LiCl}, \mathrm{LiOH}, \mathrm{Li} 2 \mathrm{O}, \mathrm{Li}_{2} \mathrm{O}_{2}$ and $\mathrm{Li}_{2} \mathrm{CO}_{3}$. 


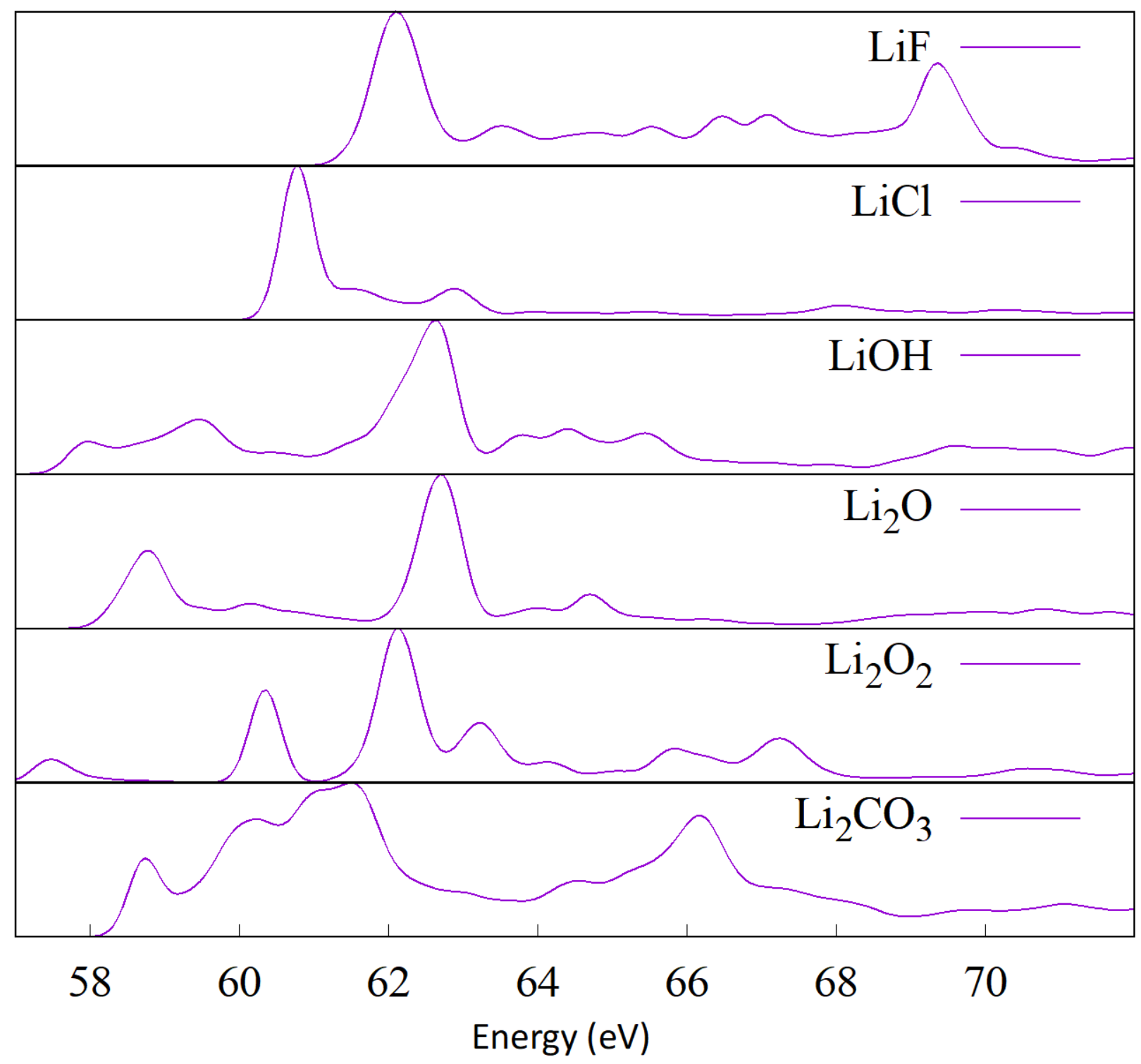

FIG.6 Li K-edge sXAS plots of $\mathrm{LiF}, \mathrm{LiCl}, \mathrm{LiOH}, \mathrm{Li}_{2} \mathrm{O}, \mathrm{Li}_{2} \mathrm{O}_{2}$ and $\mathrm{Li}_{2} \mathrm{CO}_{3}$ simulated with MBXAS. A broadening of $0.2 \mathrm{eV}$ has been applied on all spectra. For a discussion on the spectral alignment, see main text. 


\section{ASSOCIATED CONTENT}

Supporting Information: A brief account of the determinant based technique used in MBXAS, MBXAS plot of Li K-edge of Li metal using two different values for broadening, Isovalue plot of representative orbitals contributing to the different peaks in the absorption spectra of some of the Li-based compounds, Contribution from different layers in MBXAS of a Li slab, plot of X-ray attenuation length as a function of photon energy.

\section{AUTHOR INFORMATION}

\section{Corresponding Authors}

ychuang@1bl.gov, dgprendergast@1bl.gov, wlyang@lbl.gov

\section{Author Contributions}

† These authors contributed equally.

Acknowledgements: Experiments were performed at BL4.0.3 of the Advanced Light Source (ALS), a DOE Office of Science User Facility at the Lawrence Berkeley National Laboratory (LBNL). Theory effort is supported by the LDRD program at the LBNL, and facilitated by a User Program at The Molecular Foundry (TMF). Computational resources were provided by the Lawrencium and the TMF clusters (managed by the High Performance Computing Services Group, at LBNL) and by the National Energy Research Scientific Computing Center (NERSC). W.Y. acknowledges the support from the DOE Data, Artificial Intelligence, and Machine Learning at DOE Scientific User Facilities project. All the LBNL facilities are supported by the Office of Science of the US DOE under Contract No. DE-AC02- 05CH11231. We would like to thank Tod Pascal for helpful discussions.

\section{REFERENCES}

(1) Reddy, M. V.; Mauger, A.; Julien, C. M.; Paolella, A.; Zaghib, K. Brief History of Early Lithium-Battery Development. Materials 2020, 13 (8), 1884. https://doi.org/10.3390/ma13081884.

(2) Lin, D. Reviving the Lithium Metal Anode for High-Energy Batteries. Nat. Nanotechnol. 2017, 12, 13.

(3) Krauskopf, T.; Richter, F. H.; Zeier, W. G.; Janek, J. Physicochemical Concepts of the Lithium Metal Anode in Solid-State Batteries. Chem. Rev. 2020, 120 (15), 7745-7794. https://doi.org/10.1021/acs.chemrev.0c00431.

(4) Winter, M.; Barnett, B.; Xu, K. Before Li Ion Batteries. Chem. Rev. 2018, 118 (23), 1143311456. https://doi.org/10.1021/acs.chemrev.8b00422.

(5) Xu, K. Electrolytes and Interphases in Li-lon Batteries and Beyond. Chem. Rev. 2014, 114 (23), 11503-11618. https://doi.org/10.1021/cr500003w. 
(6) Nishimura, S.; Kobayashi, G.; Ohoyama, K.; Kanno, R.; Yashima, M.; Yamada, A. Experimental Visualization of Lithium Diffusion in LixFePO4. Nat. Mater. 2008, 7 (9), 707711. https://doi.org/10.1038/nmat2251.

(7) Lin, F.; Liu, Y.; Yu, X.; Cheng, L.; Singer, A.; Shpyrko, O. G.; Xin, H. L.; Tamura, N.; Tian, C.; Weng, T.-C.; Yang, X.-Q.; Meng, Y. S.; Nordlund, D.; Yang, W.; Doeff, M. M. Synchrotron XRay Analytical Techniques for Studying Materials Electrochemistry in Rechargeable Batteries. Chem. Rev. 2017, 117 (21), 13123-13186. https://doi.org/10.1021/acs.chemrev.7b00007.

(8) Qiao, R.; Chuang, Y.-D.; Yan, S.; Yang, W. Soft X-Ray Irradiation Effects of Li2O2, Li2CO3 and Li2O Revealed by Absorption Spectroscopy. PLOS ONE 2012, 7 (11), e49182. https://doi.org/10.1371/journal.pone.0049182.

(9) Braun, A.; Wang, H.; Shim, J.; Lee, S. S.; Cairns, E. J. Lithium K(1s) Synchrotron NEXAFS Spectra of Lithium-Ion Battery Cathode, Anode and Electrolyte Materials. J. Power Sources 2007, 170 (1), 173-178. https://doi.org/10.1016/j.jpowsour.2007.04.022.

(10) Wang, D.; Zuin, L. Li K-Edge X-Ray Absorption near Edge Structure Spectra for a Library of Lithium Compounds Applied in Lithium Batteries. J. Power Sources 2017, 337, 100-109. https://doi.org/10.1016/j.jpowsour.2016.10.105.

(11) Tsuji, J.; Nakamatsu, H.; Mukoyama, T.; Kojima, K.; Ikeda, S.; Taniguchi, K. Lithium K-Edge XANES Spectra for Lithium Compounds. X-Ray Spectrom. 2002, 31 (4), 319-326. https://doi.org/10.1002/xrs.577.

(12) Yogi, C.; Takamatsu, D.; Yamanaka, K.; Arai, H.; Uchimoto, Y.; Kojima, K.; Watanabe, I.; Ohta, T.; Ogumi, Z. Soft X-Ray Absorption Spectroscopic Studies with Different Probing Depths: Effect of an Electrolyte Additive on Electrode Surfaces. J. Power Sources 2014, 248, 994-999. https://doi.org/10.1016/j.jpowsour.2013.10.030.

(13) Mizota, H.; Ito, Y.; Tochio, T.; Handa, K.; Takekawa, S.; Kitamura, K. Li K-Edge XANES Spectra of Lithium Niobate and Lithium Tantalite. In AIP Conference Proceedings; AIP: Stanford, California (USA), 2007; Vol. 882, pp 508-510. https://doi.org/10.1063/1.2644575.

(14) O'Shaughnessy, C.; Henderson, G. S.; Moulton, B. J. A.; Zuin, L.; Neuville, D. R. A Li K -Edge XANES Study of Salts and Minerals. J. Synchrotron Radiat. 2018, 25 (2), 543-551. https://doi.org/10.1107/S1600577518000954.

(15) Orikasa, Y.; Furutani, J.; Yamanaka, K.; Ohta, T. Lithium K-Edge X-Ray Absorption Analysis of Lithium-Ion Battery Cathodes. 2019, No. 21, 4.

(16) Zhuo, Z.; Dai, K.; Qiao, R.; Wang, R.; Wu, J.; Liu, Y.; Peng, J.; Chen, L.; Chuang, Y.; Pan, F.; Shen, Z.; Liu, G.; Li, H.; Devereaux, T. P.; Yang, W. Cycling Mechanism of Li2MnO3: Li-CO2 Batteries and Commonality on Oxygen Redox in Cathode Materials. Joule 2021, S2542435121000817. https://doi.org/10.1016/j.joule.2021.02.004.

(17) Haensel, R.; Keitel, G.; Sonntag, B.; Kunz, C.; Schreiber, P. Photoabsorption Measurement of Li, Be, Na, Mg, and Al in the XUV Range. Phys. Status Solidi A 1970, 2 (1), 85-90. https://doi.org/10.1002/pssa.19700020110.

(18) Hightower, A.; Ahn, C. C.; Fultz, B.; Rez, P. Electron Energy-Loss Spectrometry on Lithiated Graphite. Appl. Phys. Lett. 2000, 77 (2), 238-240. https://doi.org/10.1063/1.126936.

(19) Schülke, W.; Nagasawa, H.; Mourikis, S.; Lanzki, P. Dynamic Structure of Electrons in Li Metal: Inelastic Synchrotron x-Ray Scattering Results and Interpretation beyond the 
Random-Phase Approximation. Phys. Rev. B 1986, 33 (10), 6744-6757.

https://doi.org/10.1103/PhysRevB.33.6744.

(20) Pascal, T. A.; Boesenberg, U.; Kostecki, R.; Richardson, T. J.; Weng, T.-C.; Sokaras, D.; Nordlund, D.; McDermott, E.; Moewes, A.; Cabana, J.; Prendergast, D. Finite Temperature Effects on the X-Ray Absorption Spectra of Lithium Compounds: First-Principles Interpretation of X-Ray Raman Measurements. J. Chem. Phys. 2014, 140 (3), 034107. https://doi.org/10.1063/1.4856835.

(21) Fister, T. T.; Schmidt, M.; Fenter, P.; Johnson, C. S.; Slater, M. D.; Chan, M. K. Y.; Shirley, E. L. Electronic Structure of Lithium Battery Interphase Compounds: Comparison between Inelastic x-Ray Scattering Measurements and Theory. J. Chem. Phys. 2011, 135 (22), 224513. https://doi.org/10.1063/1.3664620.

(22) Hohenberg, P.; Kohn, W. Inhomogeneous Electron Gas. Phys. Rev. 1964, 136 (3B), B864B871. https://doi.org/10.1103/PhysRev.136.B864.

(23) Kohn, W.; Sham, L. J. Self-Consistent Equations Including Exchange and Correlation Effects. Phys. Rev. 1965, 140 (4A), A1133-A1138. https://doi.org/10.1103/PhysRev.140.A1133.

(24) Rohlfing, M.; Louie, S. G. Electron-Hole Excitations and Optical Spectra from First Principles. Phys. Rev. B 2000, 62 (8), 4927-4944. https://doi.org/10.1103/PhysRevB.62.4927.

(25) Mauchamp, V.; Boucher, F.; Ouvrard, G.; Moreau, P. Ab Initio Simulation of the Electron Energy-Loss near-Edge Structures at the Li K Edge in Li, Li 20 , and Li Mn 20 4. Phys. Rev. B 2006, 74 (11), 115106. https://doi.org/10.1103/PhysRevB.74.115106.

(26) Liang, Y.; Vinson, J.; Pemmaraju, S.; Drisdell, W. S.; Shirley, E. L.; Prendergast, D. Accurate X-Ray Spectral Predictions: An Advanced Self-Consistent-Field Approach Inspired by ManyBody Perturbation Theory. Phys. Rev. Lett. 2017, 118 (9), 096402. https://doi.org/10.1103/PhysRevLett.118.096402.

(27) Liang, Y.; Prendergast, D. Quantum Many-Body Effects in x-Ray Spectra Efficiently Computed Using a Basic Graph Algorithm. Phys. Rev. B 2018, 97 (20), 205127. https://doi.org/10.1103/PhysRevB.97.205127.

(28) Yang, W. L. Band Structure and Fermi Surface of Electron-Doped C60 Monolayers. Science 2003, 300 (5617), 303-307. https://doi.org/10.1126/science.1082174.

(29) Krisch, M. H.; Sette, F.; Masciovecchio, C.; Verbeni, R. Momentum Transfer Dependence of Inelastic X-Ray Scattering from the Li K Edge. Phys. Rev. Lett. 1997, 78 (14), 2843-2846. https://doi.org/10.1103/PhysRevLett.78.2843.

(30) Jiang, P.; Prendergast, D.; Borondics, F.; Porsgaard, S.; Giovanetti, L.; Pach, E.; Newberg, J.; Bluhm, H.; Besenbacher, F.; Salmeron, M. Experimental and Theoretical Investigation of the Electronic Structure of $\mathrm{Cu}_{2} \mathrm{O}$ and $\mathrm{CuO}$ Thin Films on $\mathrm{Cu}(110)$ Using $\mathrm{x}$-Ray Photoelectron and Absorption Spectroscopy. J. Chem. Phys. 2013, 138 (2), 024704. https://doi.org/10.1063/1.4773583.

(31) England, A. H.; Duffin, A. M.; Schwartz, C. P.; Uejio, J. S.; Prendergast, D.; Saykally, R. J. On the Hydration and Hydrolysis of Carbon Dioxide. Chem. Phys. Lett. 2011, 514 (4-6), 187195. https://doi.org/10.1016/j.cplett.2011.08.063.

(32) Prendergast, D.; Galli, G. X-Ray Absorption Spectra of Water from First Principles Calculations. Phys. Rev. Lett. 2006, 96 (21), 215502. https://doi.org/10.1103/PhysRevLett.96.215502. 
(33) Mori-Sánchez, P.; Cohen, A. J.; Yang, W. Localization and Delocalization Errors in Density Functional Theory and Implications for Band-Gap Prediction. Phys. Rev. Lett. 2008, 100 (14), 146401. https://doi.org/10.1103/PhysRevLett.100.146401.

(34) Perdew, J. P.; Yang, W.; Burke, K.; Yang, Z.; Gross, E. K. U.; Scheffler, M.; Scuseria, G. E.; Henderson, T. M.; Zhang, I. Y.; Ruzsinszky, A.; Peng, H.; Sun, J.; Trushin, E.; Görling, A. Understanding Band Gaps of Solids in Generalized Kohn-Sham Theory. Proc. Natl. Acad. Sci. 2017, 114 (11), 2801-2806. https://doi.org/10.1073/pnas.1621352114.

(35) Perdew, J. P.; Burke, K.; Ernzerhof, M. Generalized Gradient Approximation Made Simple. Phys. Rev. Lett. 1996, 77 (18), 3865-3868. https://doi.org/10.1103/PhysRevLett.77.3865.

(36) Heyd, J.; Scuseria, G. E.; Ernzerhof, M. Hybrid Functionals Based on a Screened Coulomb Potential. J. Chem. Phys. 2003, 118 (18), 8207-8215. https://doi.org/10.1063/1.1564060.

(37) Heyd, J.; Scuseria, G. E. Efficient Hybrid Density Functional Calculations in Solids: Assessment of the Heyd-Scuseria-Ernzerhof Screened Coulomb Hybrid Functional. J. Chem. Phys. 2004, 121 (3), 1187-1192. https://doi.org/10.1063/1.1760074.

(38) Janesko, B. G.; Henderson, T. M.; Scuseria, G. E. Screened Hybrid Density Functionals for Solid-State Chemistry and Physics. Phys Chem Chem Phys 2009, 11 (3), 443-454. https://doi.org/10.1039/B812838C.

(39) Giannozzi, P.; Baroni, S.; Bonini, N.; Calandra, M.; Car, R.; Cavazzoni, C.; Ceresoli, D.; Chiarotti, G. L.; Cococcioni, M.; Dabo, I.; Dal Corso, A.; de Gironcoli, S.; Fabris, S.; Fratesi, G.; Gebauer, R.; Gerstmann, U.; Gougoussis, C.; Kokalj, A.; Lazzeri, M.; Martin-Samos, L.; Marzari, N.; Mauri, F.; Mazzarello, R.; Paolini, S.; Pasquarello, A.; Paulatto, L.; Sbraccia, C.; Scandolo, S.; Sclauzero, G.; Seitsonen, A. P.; Smogunov, A.; Umari, P.; Wentzcovitch, R. M. QUANTUM ESPRESSO: A Modular and Open-Source Software Project for Quantum Simulations of Materials. J. Phys. Condens. Matter 2009, 21 (39), 395502. https://doi.org/10.1088/0953-8984/21/39/395502. 


\title{
SUPPLEMENTARY INFORMATION
}

\section{Controlled Experiments and Optimized Theory of Absorption Spectra of Li Metal and Salts}

\author{
Subhayan Roychoudhury ${ }^{\S \dagger}$, Zengqing Zhuo ${ }^{\S \dagger}$, Ruimin Qiao ${ }^{\S \dagger}$, Liwen Wan ${ }^{\text {II }}$, Yufeng Liang ${ }^{\#}$, Feng \\ Pan $^{\ddagger}$, Yi-de Chuang ${ }^{\S *}$, David Prendergast ${ }^{\# *}$, Wanli Yang ${ }^{{ }^{*}}$ \\ ${ }^{\S}$ Advanced Light Source, Lawrence Berkeley National Laboratory, 1 Cyclotron Road, Berkeley \\ CA 94720, USA \\ "Lawrence Livermore National Laboratory, 7000 East Avenue, Livermore, California 94550, USA \\ ${ }^{\ddagger}$ School of Advanced Materials, Peking University Shenzhen Graduate School, Shenzhen \\ 518055, China \\ \#The Molecular Foundry, Lawrence Berkeley National Laboratory, 1 Cyclotron Road, Berkeley \\ CA 94720, USA
}

\section{Determinant based technique used in MBXAS}

Simulations of Li K-edge spectra are performed with the MBXAS software, which uses the energy and Kohn-Sham orbitals calculated with the DFT-based basis code Quantum Espresso ${ }^{39}$. Since a core-excited state calculation involves an atom containing one less core electron (represented by the appropriate pseudopotential), electronic structure calculations were performed with large supercells to minimize the effects of interaction among the periodic replica. Bulk Li simulations were performed with a cubic supercell of length $\sim 26$ a.u. For simulation of the (100) Li slab, a vacuum of $\sim 36$ a.u. is added above the surface layer. DFT calculations were performed employing ultrasoft pseudopotentials and a plane-wave cutoff-energy of $25 \mathrm{Ry}$ (200Ry) is used to represent the wave-functions (charge density). All calculations on Li metal (bulk and slab) have been performed with the parameter "maxfn=2", while those on the compounds have been performed with "maxfn=1". In a calculation with $\operatorname{maxfn}=M$, the code can create up to $(M-1)$ number of electron-hole pairs in the valence subspace in addition to the core-excitation. Typically, a calculation with maxfn $>1$ is deemed necessary only for metals and small-gap semiconductors because of the low energy required to create a valence excitation.

In MBXAS, the transition matrix elements are expressed as:

Where $\left\langle\Psi_{\mathrm{f}} \mid \Psi_{i}^{c}\right\rangle$ follows the relation

$$
\left\langle\Psi_{\mathrm{f}}|\widehat{\mathbf{0}}| \Psi_{\mathrm{i}}\right\rangle=\sum_{c}^{\text {empty }}\left\langle\Psi_{\mathrm{f}} \mid \Psi_{i}^{c}\right\rangle\left\langle\phi_{c}|\hat{\mathbf{o}}| \phi_{\text {core }}\right\rangle
$$




$$
\left\langle\Psi_{\mathrm{f}} \mid \Psi_{i}^{c}\right\rangle^{*}=\operatorname{det}\left[\begin{array}{ccccc}
\xi_{\tilde{f}_{1}, 1} & \xi_{\tilde{f}_{1}, 2} & \cdots & \xi_{\tilde{f}_{1}, N} & \xi_{\tilde{f}_{1}, c} \\
\vdots & \vdots & \ddots & \vdots & \vdots \\
\xi_{\tilde{f}_{N+1}, 1} & \xi_{\tilde{f}_{N+1}, 2} & \cdots & \xi_{\tilde{f}_{N+1}, N} & \xi_{\tilde{f}_{N+1}, c}
\end{array}\right] .
$$

Here, $\left\{\tilde{f}_{1}, \tilde{f}_{2}, \cdots, \tilde{f}_{N+1}\right\}$ is the set of occupied final-state orbitals (obtained with an SCF calculation employing pseudopotential containing core-hole) constituting $\Psi_{\mathrm{f}}$ and $\xi_{\tilde{f}_{l}, m}=\left\langle\phi_{m} \mid \widetilde{\phi_{\tilde{f}_{l}}}\right\rangle$, where a $\sim$ on top denotes a final state orbital index/wave-function. FIG $4 \mathrm{C}$ in the main text shows, in the negative log scale, the heat-map of the elements of the matrix $\xi$. 


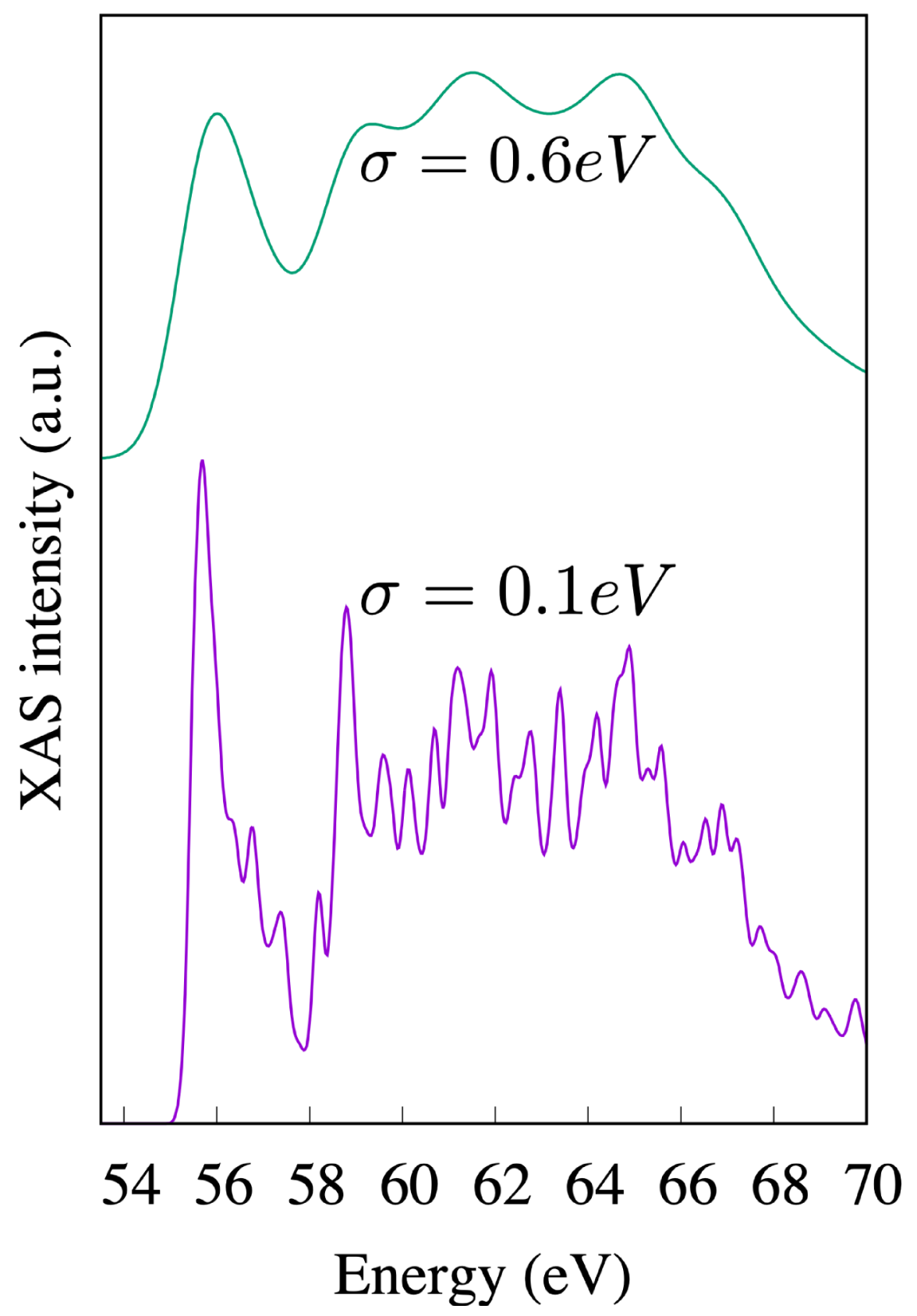

FIG S1: MBXAS plot of Li K-edge sXAS of pristine bulk Li for two different values of broadening, $0.1 \mathrm{eV}$ and $0.6 \mathrm{eV}$. 


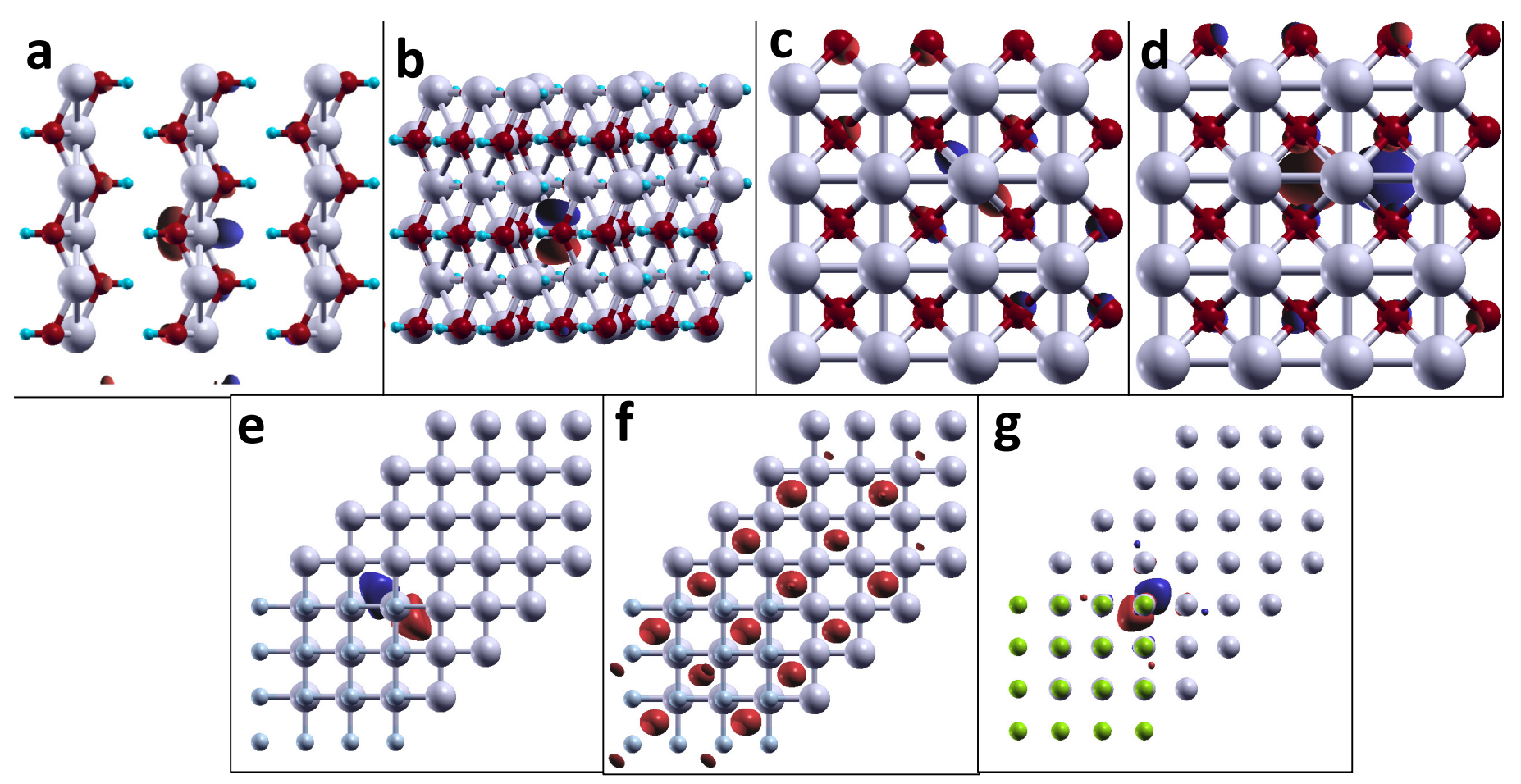

FIG S2: Isovalue plot of representative orbitals contributing to the different peaks in the absorption spectra of some of the Li-based compounds. (a) Peak at $\sim 59.5 \mathrm{eV}$ for $\mathrm{LiOH}$, (b) peak at $\sim 61.6 \mathrm{eV}$ for $\mathrm{LiOH}$, (c) peak at $\sim 58.8 \mathrm{eV}$ for $\mathrm{Li}_{2} \mathrm{O}$, (d) peak at $\sim 62.7 \mathrm{eV}$ for $\mathrm{Li}_{2} \mathrm{O}$, (e) peak at $\sim 62.1 \mathrm{eV}$ for $\mathrm{LiF}$, (f) peak at $\sim 69.4 \mathrm{eV}$ for $\mathrm{LiF},(\mathrm{g})$ peak at $\sim 60.8 \mathrm{eV}$ for $\mathrm{LiCl}$. 


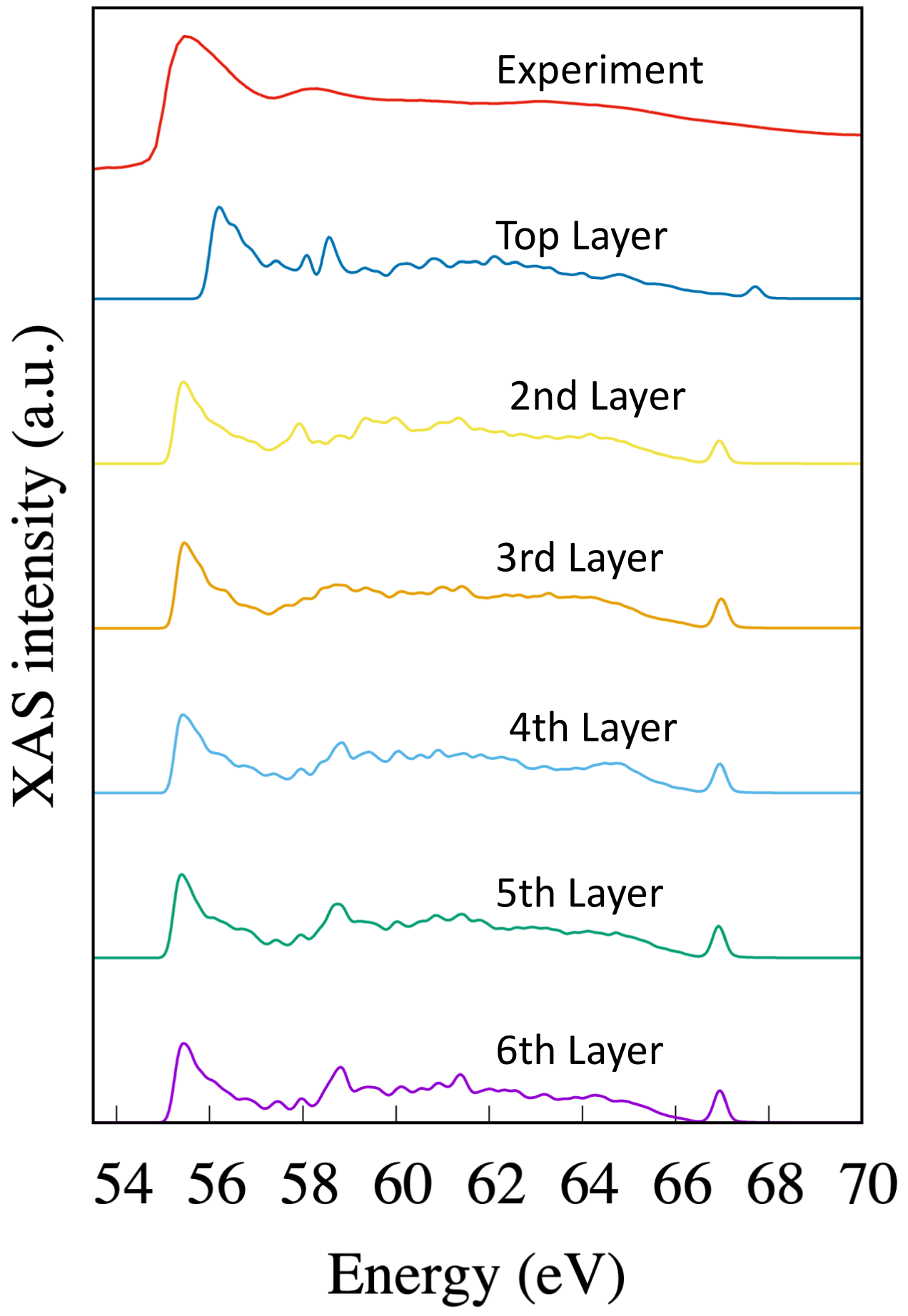

FIG S3: Simulated sXAS plots of the top (blue), $2^{\text {nd }}$ (yellow), $3^{\text {rd }}$ (orange), $4^{\text {th }}$ (indigo), $5^{\text {th }}$ (green) and $6^{\text {th }}$ (violet) layers of the 12-layer thick Li slab. The experimental plot (red) is presented on top. 


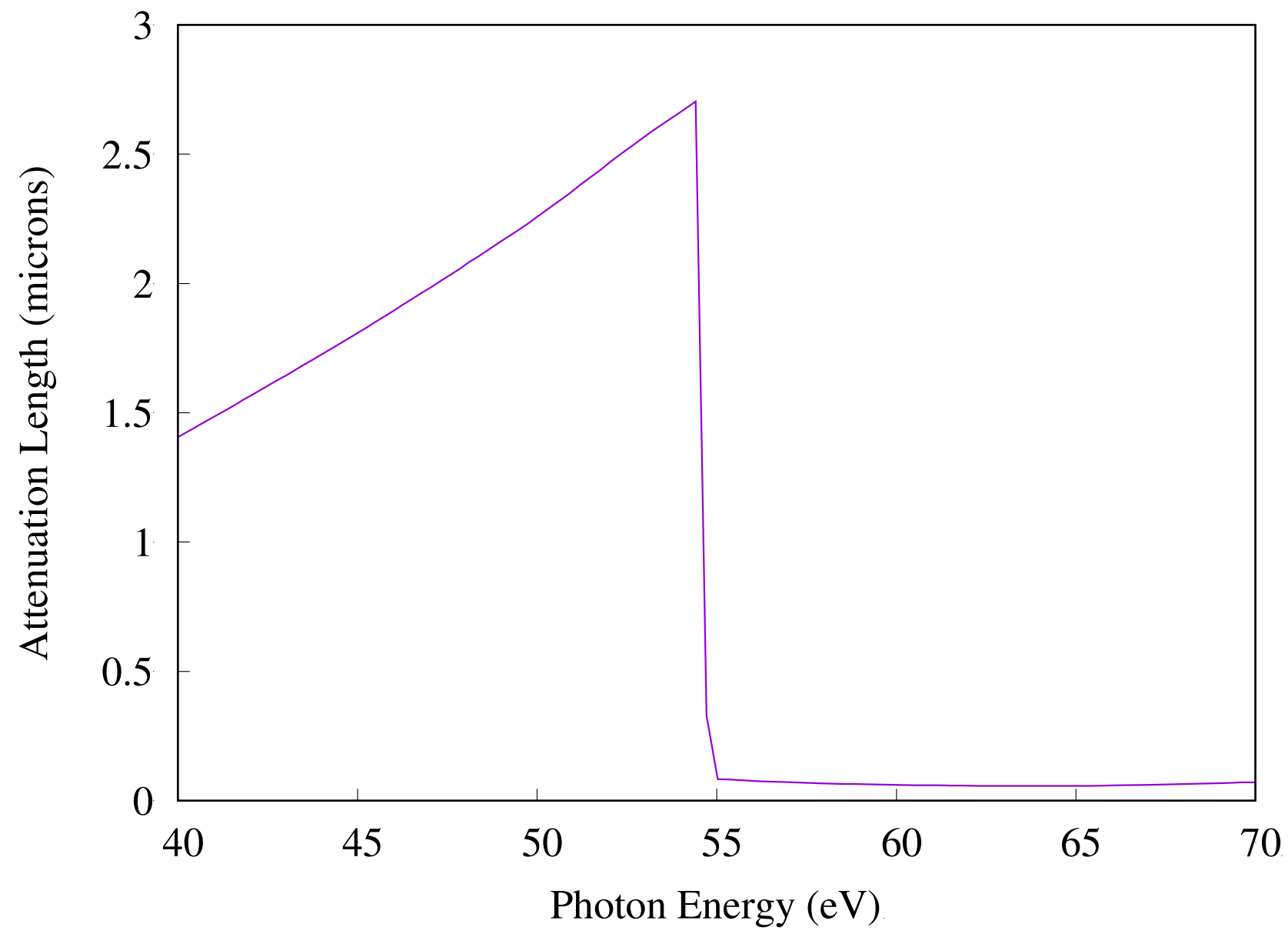

FIG S4: Plot of X-ray attenuation length as a function of photon energy incident at 90. Deg angle on Li metal. 